\title{
Searching for dark sector with missing mass technique in fixed target experiments
}

\author{
Venelin Kozhuharov ${ }^{1,2, *}$ \\ ${ }^{1}$ Faculty of Physics, University of Sofia, 5 J. Bourchier Blvd., BG-1164 Sofia, Bulgaria \\ ${ }^{2}$ Laboratori Nazionali di Frascati, IT-00044 Frascati (RM), Italy
}

\begin{abstract}
Currently, the existence of a dark sector almost completely decoupled from the Standard Model is a viable solution for numerous long-standing problems in physics, including the nature of dark matter and the muon anomalous magnetic moment. A new gauge mediator, the dark photon, could be the portal to this hidden sector. The most general probe to its existence is the missing mass technique which requires a precise knowledge of the initial state of the process but does not put constraints on the dark photon final states. The experimental approaches to the search for dark photons in positron-on-target annihilation and in mesons decay in flight are presented and the physics reach is discussed.
\end{abstract}

\section{Introduction}

Despite its great success, the Standard Model of particle physics does not provide a viable explanation for several astrophysics phenomena, including the matter-antimatter asymmetry in the Universe and the existence and the abundance of Dark matter. There are also few experimental signatures in tension with the Standard Model (SM) predictions - the measured anomalous magnetic moment of the muon $\left(g_{\mu}-2\right)$ [1] and the indications for the possible existence of a new light neutral boson in the deexcitation of ${ }^{8} B e^{*}[2]$.

The aforementioned experimental observables could be addressed with the introduction of a new sector of particles which do not exhibit $S U(3)_{C} \times S U(2)_{L} \times U(1)_{Y}$ quantum numbers, making it neutral for the known gauge interactions. The connection between the two sectors, the Standard Model and the hidden one, is realized through a portal - one or limited number of particles that interact with states from both the visible and the hidden sector.

Depending on the properties of the mediating particle several realization of the portal are generally considered - vector portal, higgs portal, neutrino portal, scalar portal.

One of the simplest possible realization of the vector portal [3], [4] is the introduction of a vector gauge field, the so-called Dark photon (DP) $A^{\prime}$, which interacts weakly with the SM fermions

$$
\mathcal{L} \sim g^{\prime} q_{f} \bar{\psi}_{f} \gamma^{\mu} \psi_{f} A_{\mu}^{\prime},
$$

where $g^{\prime}$ is the universal coupling constant and $q_{f}$ are the corresponding fermion charges. The term in equation (1) could be effectively realized also through the so called kinetic mixing of the massive DP with the ordinary photon. Then, the interaction of the SM particles with

\footnotetext{
*e-mail: venelin@phys.uni-sofia.bg
} 
the Dark photon will be described by only two parameters - $\epsilon \sim g^{\prime} q_{f}$ and the dark photon mass $m_{A^{\prime}}$. The coupling parameter $\epsilon$ could also be flavour dependent [5]. The existence of a vector particle with such characteristics may also contribute to the anomalous magnetic moment of the muon [6] and be the origin of the present discrepancy between the theory and the experiment [1].

The dark sector may also contain additional degrees of freedom, $\chi \mathrm{s}$ with mass $m_{\chi}$, which couple to $A^{\prime}$ with a relative coupling strength $\alpha_{D}$. Depending on the mass hierarchy between $\chi$ and $A^{\prime}$, the phenomenology of the events with Dark photon could be divided into two distinctive scenarios.

- If $m_{\chi}>m_{A^{\prime}}$ then the Dark photon decays to SM particles with a rate proportional to $\epsilon^{2}$. For $m_{A^{\prime}}<2 m_{\mu}$ the only allowed final state is $e^{+} e^{-}$.

- If $\chi$ is light and $m_{A^{\prime}}>2 m_{\chi}$ then the dominant decay process will be $A^{\prime} \rightarrow \chi \chi$ since it is not suppressed by the small factor $\epsilon$.

\section{Searching for Dark Photons in constraint initial state processes}

Two complementary paths could be followed when searching for new states - the so called energy and intensity frontiers. While the former usually exploits collider technique and gives access to high masses, the latter addresses the extremely small coupling constants scenarios through high rate experimental setups in beam-on-target technique.

\subsection{Primary proton beam}

In proton-on-target experiments the missing mass searches for Dark photon can be performed in meson decays. This could be done either in the decays of mesons directly produced at the target or in the decays of mesons which are decay products of fully reconstructable state.

In the case of the kinetic mixing scenario, the branching fraction for the decay $M \rightarrow X A^{\prime}$ is scaled with respect to the $B R(M \rightarrow X \gamma)$ by a factor of $\varepsilon^{2} \times f\left(m_{A^{\prime}}\right)$, where $f\left(m_{A^{\prime}}\right)$ is a kinematics factor and $X=X_{1}+X_{2}+\ldots$. The missing mass is then given by $M_{\text {miss }}=$ $\left(P_{M}-\sum_{i} P_{X_{i}}\right)^{2}$ and requires the detection of all particles $X_{i}$.

The necessity to know precisely the momentum of the decaying meson $P_{M}$ limits the possible choices for directly produced in the target $M$ to sufficiently longer living pions and kaons, which can be selected through a system of achromats. In the case of kaons, of interest is the FCNC process $K^{ \pm} \rightarrow \pi^{ \pm} A^{\prime}$, with $A^{\prime} \rightarrow$ invisible, which has the same experimental signature as $K^{ \pm} \rightarrow \pi^{ \pm} v \bar{v}$.

The Dark photon can also be produced in the decays of the neutral pions which themselves are product of kaon decays. The probability for $\pi^{0} \rightarrow \gamma A^{\prime}$ is given by

$$
\operatorname{Br}\left(\pi^{0} \rightarrow \gamma A^{\prime}\right)=2 \varepsilon^{2}\left(1-\frac{m_{A^{\prime}}^{2}}{m_{\pi^{0}}^{2}}\right)^{3} \times B r\left(\pi^{0} \rightarrow \gamma \gamma\right)
$$

The initial $K \rightarrow \pi^{0} X$ decay allows the determination of the $\pi^{0}$ momentum and the closure of the kinematics.

\subsection{Primary positron beam}

The positron-on-target technique was addressed only recently but it has the potential to increase the precision ot the studies. The basic idea is schematically shown in fig. 1 and relies 


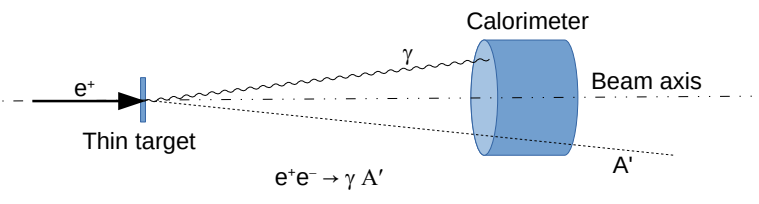

Figure 1. Searching for Dark photon with missing mass technique in positron-on-target setup.

on the production of a Dark photon in the annihilation of the beam positrons with the atomic electrons.

The primary positron beam is with well defined characteristics, depending on the employed accelerator. The cross section for Dark photon production is related to the cross section for two photon annihilation through

$$
\sigma\left(e^{+} e^{-} \rightarrow \gamma A^{\prime}\right)=2 \varepsilon^{2} \sigma\left(e^{+} e^{-} \rightarrow \gamma \gamma\right) \times \delta^{\prime},
$$

where $\delta^{\prime}$ is the kinematics enhancement factor which equals to 1 when $\sqrt{s}>>M_{A^{\prime}}$. The missing mass is given as $M_{\text {miss }}=\left(P_{e^{+}}+P_{e^{-}}-P_{\gamma}\right)^{2}$, with $P_{e^{-}}=\left(m_{e}, 0,0,0\right)$ in most of the cases. The recoil photon energy and impact point are measured by an electromagnetic calorimeter.

\section{Dark Photon in $\pi^{0}$ decays at NA62}

A search for Dark photon produced in $\pi^{0}$ decays was performed at the NA62 experiment, schematically shown in fig. 2 [7]. The primary goal of NA62 is the measurement of the $\operatorname{Br}\left(K^{+} \rightarrow \pi^{+} v \bar{v}\right)$ with $10 \%$ precision [8].

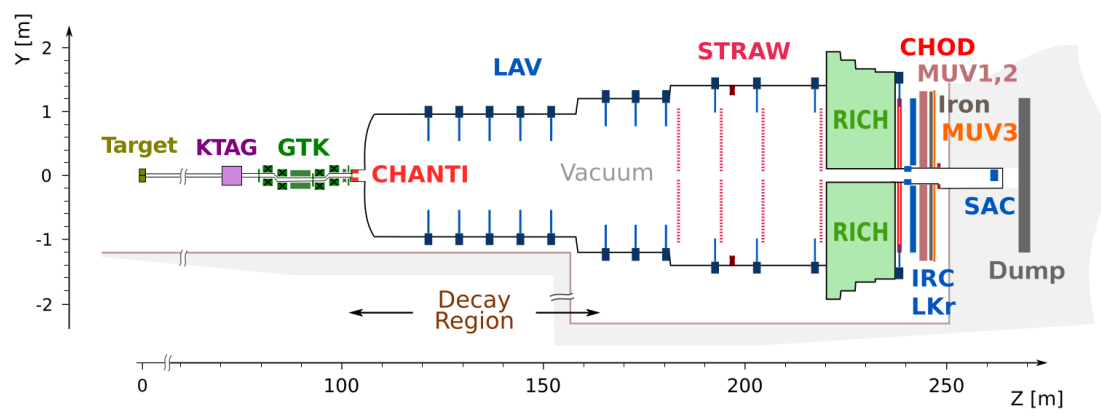

Figure 2. Schematics of the NA62 experiment at CERN SPS.

Kaons are produced in the interaction of the $400 \mathrm{GeV} / \mathrm{c}$ primary proton beam from CERN SPS on a beryllium target. They are selected with a nominal momentum of $75 \mathrm{GeV} / \mathrm{c}$ and RMS of $1 \%$. The kaons are positively identified by a differential Cherenkov counter (KTaG) and their momentum and direction are measured by the GTK. The charged decay products momentum is measured by a straw-tube spectrometer (STRAW), accompanied by a ring imaging Cherenkov detector for pion identification. A fast scintillator hodoscope (CHOD) is used for timing. The photons are detected by a hermetic photon system composed by two 
lead-scintillator sandwich detectors (SAC and IRC), a liquid krypton calorimeter (LKr), and a system of 12 annular lead-glass detectors. The muon suppression was further enhanced by a muon veto system (MUV). The high kaon flux combined with precise kinematics measurement, particle identification, and hermeticity make the NA62 detector extremely powerful for the study of rare processes.

A partial data set recorded in 2016 was used to look for the $K^{+} \rightarrow \pi^{+} \pi^{0}, \pi^{0} \rightarrow \gamma A^{\prime}$ events. No statistically significant signal was observed. The preliminary studies and results, shown in fig. 4, indicated that NA62 is sensitive to the mass range $30 \mathrm{MeV} / \mathrm{c}^{2}<M_{A^{\prime}}<120 \mathrm{MeV} / \mathrm{c}^{2}$ and is competitive and complementary to other searches.

\section{Invisible Dark photon at PADME}

The PADME experiment [9] at LNF-INFN realizes the technique described in sec. 2.2. The DAФNE linac provides $550 \mathrm{MeV}$ positrons [10],[11] grouped in 49 bunches per second, each with $\sim 200$ ns duration. The optimal number of particles per bunch is determined by the rate limitations of the PADME subdetectors and amounts to $\sim 20000 e^{+}$.

The positrons are directed towards a $100 \mu \mathrm{m}$ thick diamond target with transverse dimensions of $20 \times 20 \mathrm{~mm}^{2}$ [12]. $1 \mathrm{~mm}$ wide graphitized strips realized with excimer ArF laser [13] and oriented orthogonality on the target sides measure the beam impact position and the positron multiplicity. The linearity of the target response was verified up to $\sim 30000$ positrons in a single bunch.

The PADME calorimetric system is composed of two crystal calorimeters - a ring shaped main calorimeter (ECAL [14]) with diameter $\sim 60 \mathrm{~cm}$, made of 616 BGO crystals and a Small Angle Calorimeter (SAC [15]) covering the inner hole of the ECAL and based on the detection of the Cerenkov light produced from the interactions of high energy particles in a $\mathrm{PbF}_{2}$ crystal. Each of the BGO-PMT assemblies were calibrated using a ${ }^{22} \mathrm{Na}$ source and the expected energy resolution of the ECAL is $\sigma(E) / E=2.0 \% / \sqrt{E} \oplus 1.1 \%$ (E is in $\mathrm{GeV}$ ).

The non-interacted positron beam is taken outside the acceptance of the calorimeters by a dipole magnet providing $0.6 \mathrm{~T}$ field over length of $\mathrm{O}(1 \mathrm{~m})$. Three station of plastic scintillator based charged particle detectors are placed inside the vacuum chamber of the experiment. They detect the positrons that have radiated a high energy photon in the target (PVeto), electrons from the beam interactions in the target or from particles which decay to final states with electrons (EVeto), and the positrons with a relatively low energy radiation in the target (HEPVeto) [17]. The scintillating light is taken by BCF-92 WLS fibers to S13360 Hamamatsu SiPMs. The detector with the custom developed front end electronics provide efficiency better than $99 \%$ and time resolution better than $1 \mathrm{~ns}$ [16].

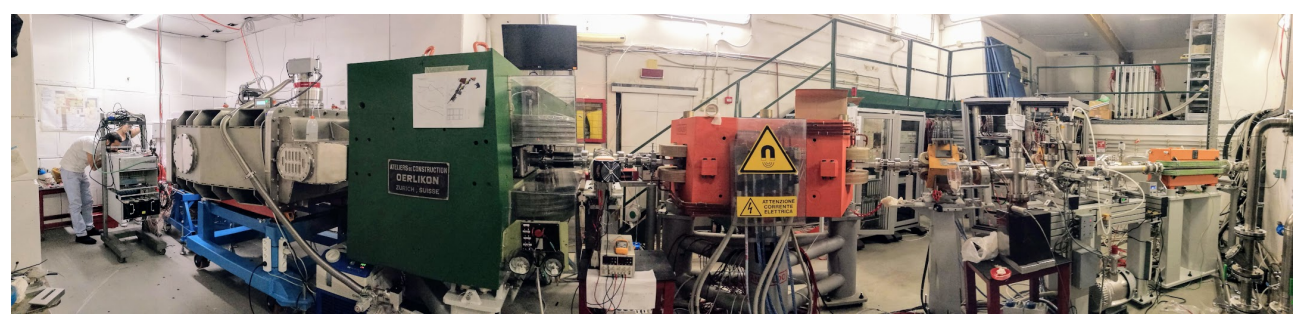

Figure 3. A photograph of the PADME beam line and experimental setup.

The dominant backgrounds to the search for Dark photon [18] are the multiphoton annihilation $\left(e^{+} e^{-} \rightarrow \gamma \gamma(\gamma)\right)$, suppressed by the calorimetry system and the bremsstrahlung 
emission from the positrons in the target $\left(e^{+} N \rightarrow e^{+} N \gamma\right)$, suppressed by the charged particle detectors.

A detailed GEANT4 [19] based simulation of the experimental apparatus was developed to study the sensitivity to the Dark photons. The expected $e^{+} e^{-} \rightarrow \gamma A^{\prime}$ yield as a function of $\epsilon$ and $M_{A^{\prime}}$ was obtained from CalcHEP [20] with the implemented lagrangian term from eq. 1. Assuming collection of $4 \times 10^{13}$ positrons, the expected sensitivity limits to the Dark photon parameters $\epsilon$ and $M_{A^{\prime}}$ is given on Fig. 4. PADME covers the region down to $\epsilon \sim 5 \times 10^{-7}$ up to the kinematical limit of $\sim 23 \mathrm{MeV}$ on the Dark photon mass.

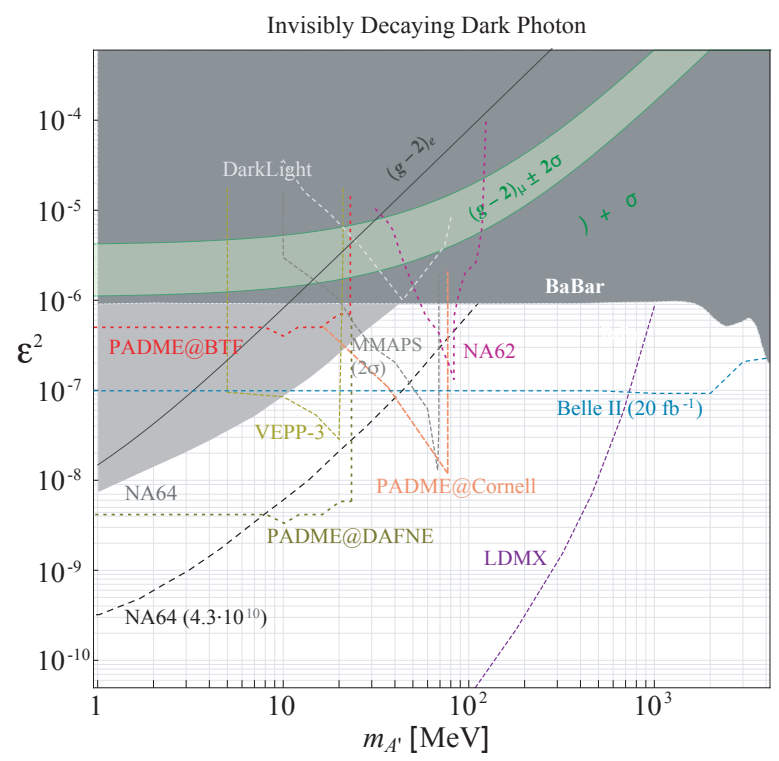

Figure 4. Sensitivity of various experiments to invisibly decaying Dark Photon. One should note that different experimental techniques are combined on the same plot.

PADME setup assembly, shown in fig. 3, was completed in summer 2018, data taking started in autumn 2018, and during the first phase about 270 TB of data were recorded in three effective months, reaching about $30 \%$ of the planned positron-on-target statistics. The detector performance studies, calibration, and analysis are ongoing. The constructed setup is universal for the study of new light particles, including also scalar particles [21].

\section{Conclusions and prospects}

The present world charged kaon factory is the NA62 experiment at CERN SPS. Its plans are to collect more than $O\left(10^{13}\right)$ kaon decays in the fiducial region which will allow a sensitive study of invisible Dark photon, probing also leptophobic scenarios.

On the leptophilic side, the only experiment searching for new light particles with positron-on-target technique is PADME. Its sensitivity is limited by two factors - the maximal positron beam energy sets an upper value for the accessible mass range in the annihilation process of $23 \mathrm{MeV}$ while the beam intensity and the duty factor sets the minimal accessible relative coupling strength $\varepsilon^{2}$.

The maximal achievable mass can be increased with the MMAPS experiment [22] by using positron beam at Cornell, extracted slowly from the synchrotron with energy of the 
order of $6 \mathrm{GeV}$. This gives limit $m_{A^{\prime}} \sim 75 \mathrm{MeV} / \mathrm{c}^{2}$. The annihilation events rate can be increased with the search technique proposed in [23]. It suggests to construct a by-pass on the current VEPP3-3 storage ring and use a hydrogen gas directly injected in the beam region as target. This could push the sensitivity to $\varepsilon^{2}$ down to $10^{-8}$. In addition, the present DAФNE collider could be converted for slow positron extraction within the POSEYDON facility [24], allowing to increase the PADME duty factor by at least three orders of magnitude.

\section{Acknowledgements}

The author acknowledges partial support by BG-NSF under grant DN-08/14 from 14.12.2016 and MoU SU - LNF-INFN 70-06-497/07-10-2014.

\section{References}

[1] G. W. Benett et al. (The g-2 Collaboration), Phys. Rev. D 73, 072003 (2006).

[2] A. J. Krasznahorkay et al., Phys. Rev. Lett. 116, no. 4, 042501 (2016).

[3] B. Holdom, Phys. Lett. B 166, 196 (1986).

[4] J. Alexander et al., arXiv:1608.08632 [hep-ph] (2016).

[5] M. Raggi and V. Kozhuharov, Riv. Nuovo Cim. 38, no. 10, 449 (2015).

[6] M. Pospelov, Phys. Rev. D 80, 095002 (2009).

[7] E. Cortina Gil et al. [NA62 Collaboration], JINST 12, no. 05, P05025 (2017).

[8] E. Cortina Gil et al. [NA62 Collaboration], Phys. Lett. B 791, 156 (2019).

[9] M. Raggi and V. Kozhuharov, Adv. High Energy Phys. 2014, 959802 (2014).

[10] A. Ghigo et al., Nucl. Instrum. Meth. A 515, 524 (2003).

[11] P. Valente et al., arXiv:1603.05651 [physics.acc-ph] (2016).

[12] G. Chiodini [PADME group], JINST 12, no. 02, C02036 (2017).

[13] M. De Feudis et al., Diam. Relat. Mater. 75, 25 (2017).

[14] M. Raggi et al., Nucl. Instrum. Meth. A 862, 31 (2017).

[15] A. Frankenthal et al., Nucl. Instrum. Meth. A 919, 89 (2019).

[16] G. Georgiev et al. IEEE Trans. Nucl. Science 65 (2018) 2029.

[17] S. Ivanov et. al, AIP Conf. Proc. 2075 (2019) no.1, 080005.

[18] V. Kozhuharov, EPJ Web Conf. 142, 01018 (2017).

[19] E. Leonardi, V. Kozhuharov, M. Raggi and P. Valente, J. Phys. Conf. Ser. 898, no. 4, 042025 (2017).

[20] A.Pukhov et al., Preprint INP MSU 98-41/542 arXiv:hep-ph/9908288 (1999);

A.Pukhov, arXiv:hep-ph/0412191 (2004).

[21] M. Raggi, V. Kozhuharov and P. Valente, EPJ Web Conf. 96, 01025 (2015).

[22] J. Alexander, EPJ Web Conf. 142, 01001 (2017).

[23] I. Rachek, D. Nikolenko and B. Wojtsekhowski, EPJ Web Conf. 142 (2017) 01025.

[24] P. Valente, arXiv:1711.06877 [physics.acc-ph]. 